\title{
Oral fingolimod to treat multiple sclerosis: see your cardiologist first
}

Fingolimode oral para tratamento da esclerose múltipla: consulte o seu cardiologista antes Douglas Kazutoshi Sato ${ }^{1,2}$, Dagoberto Callegaro²

${ }^{1}$ Department of Neurology, Tohoku University School of Medicine, Sendai, Japan;

${ }^{2}$ Department of Neurology, Faculty of Medicine, University of Sao Paulo, Sao Paulo, Brazil.

Correspondence:

Douglas Kazutoshi Sato; Department of Neurology, Tohoku University

School of Medicine. 1-1, Seiryomachi, Aobaku, Sendai, Miyagi;

980-8574, Japan;

E-mail:douglas.sato@med.tohoku. ac.jp

Conflict of interest:

Dr. Sato has received speaker

honoraria from Novartis.

Received 01 August 2014 Accepted 21 August 2014

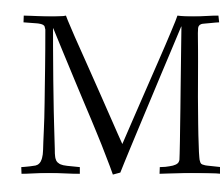

ultiple sclerosis (MS) is a chronic inflammatory demyelinating central nervous system that affects more than 2 million people worldwide ${ }^{1}$. There are strong evidences that the immune system is involved in MS physiopathology, but the exact mechanism is yet unknown. The risk of developing MS is influenced by a number of genetic and environmental factors. MS patients with a relapsing-remitting disease may have a lack of response to treatments associated or not with neutralizing antibodies $^{2}$, limitations to use injectable drugs or show intolerance to first line immunomodulatory drugs (interferon-beta $1 \mathrm{a}$ or $1 \mathrm{~b}$, and glatiramer acetate). Some of them may evolve to secondary progressive MS after some years, showing signs of neurodegeneration like increased disability, cognitive dysfunction and loss of quality of life. Therefore, other options to treat MS are required to reduce the number of relapses, but also the risk of permanent disability.

Fingolimod (also known as FTY-720) is the first oral drug that has shown efficacy in relapsing $\mathrm{MS}^{3,4}$, but further studies are required to evaluate if it has any effect on the progressive forms of MS. Fingolimod is an oral sphingosine-1-phosphate (S1P) receptor modulator that blocks lymph node egress of lymphocytes expressing the homing receptor CCR7 that may include autoreactive $\mathrm{T}$ and $\mathrm{B}$ cell subsets, and patients become gradually lymphopenic after a few days of treatment ${ }^{5}$. However, the effects of fingolimod are not limited to lymphocyte trafficking, as S1P receptors are expressed by other cells like cardiac myocytes ${ }^{6}$ and glial cells (astrocytes and oligodendrocytes $)^{7,8}$, and may promote physiological changes and activation of downstream signaling yet to be fully clarified.

In this issue of Arquivos de Neuropsiquatria, Dr. Fragoso et al. ${ }^{9}$ evaluated cardiovascular complications in 180 Brazilian MS patients during the first dose of fingolimod due to transitory effects in S1P receptors expressed in the cardiac myocytes. The severe bradycardia in $6.7 \%$ $(12 / 180)$ and atrioventricular block in 1.7\% (3/180) seen during fingolimod initiation is within the frequency found in other studies ${ }^{3,4}$. Elderly MS patients and those with history of systemic hypertension, and/or under beta-blocker treatment may be at increased risk of experiencing cardiovascular complications during the initiation of fingolimod. Nevertheless, the current worldwide post-marketing experience indicate that most patients are able to initiate safely fingolimod as long as the cardiac evaluation and monitoring is properly performed, notwithstanding the same procedures are required if the patient interrupts the drug for more than 2 weeks.

These cardiovascular effects during fingolimod initiation illustrate that adverse events from new drugs may be completely different from the first line immunomodulatory treatments for MS that have been already on the market for 15-20 years. Other known potential risks during fingolimod therapy are the development of macular edema requiring ophthalmologic examinations, increased risk of severe opportunistic infections, and development of skin cancer. However, other rare or unexpected treatment complications might arouse after a large number of patients are exposed to the drug, which were not seen during the clinical development phase. For example, some patients with neuromyelitis optica (NMO) who were unintentionally treated with fingolimod may develop severe attacks ${ }^{10}$, raising the importance of distinguishing MS from other diseases with distinct underlying pathological mechanisms.

In the past few years, fingolimod and other two oral drugs with positive results on clinical trials (teriflunomide and dimethyl fumarate), as well as monoclonal antibodies (natalizumab, 
alemtuzumab) are quickly broadening the treatment options for MS, but the complexity related to the therapeutic management of MS patients is increasing exponentially. As we do not have established biomarkers to determine which drug is the best one for each patient diagnosed with MS, answering this question currently relies on careful clinical and imaging follow-up of patients by experienced MS centers, and deep knowledge about efficacy and safety of each drug that may include clinical trials or post-marketing experience.

At least for fingolimod initiation in MS patients, an important take home message of Dr. Fragoso's study was to work closely with cardiologists and intensive care unit physicians, so any cardiovascular complications can be managed properly. As we all learned in the medical school, primum non nocere.

\section{References}

1. Milo R, Kahana E. Multiple sclerosis: geoepidemiology, genetics and the environment. Autoimmun Rev 2010;9:A387-394.

2. Sato DK, Nakashima I, Fukazawa T, et al. Neutralizing antibodies are associated with a reduction of interferon-beta efficacy during the treatment of Japanese multiple sclerosis patients. Tohoku J Exp Med 2012;228:85-92.

3. Cohen JA, Barkhof F, Comi G, et al. Oral fingolimod or intramuscular interferon for relapsing multiple sclerosis. $N$ Engl $J$ Med 2010;362:402-415.

4. Kappos L, Radue EW, O'Connor P, et al. A placebo-controlled trial of oral fingolimod in relapsing multiple sclerosis. $N$ Engl $\mathrm{J}$ Med 2010;362:387-401.

5. Sato DK, Nakashima I, Bar-Or A, et al. Changes in Th17 and regulatory $T$ cells after fingolimod initiation to treat multiple sclerosis. J Neuroimmunol 2014;268:95-98.
6. Means CK, Brown JH. Sphingosine-1-phosphate receptor signalling in the heart. Cardiovasc Res 2009;82:193-200.

7. Choi JW, Gardell SE, Herr DR, et al. FTY720 (fingolimod) efficacy in an animal model of multiple sclerosis requires astrocyte sphingosine 1-phosphate receptor 1 (S1P1) modulation. Proc Natl Acad Sci U S A 2011;108:751-756.

8. Miron VE, Jung CG, Kim HJ, Kennedy TE, Soliven B, Antel JP. FTY720 modulates human oligodendrocyte progenitor process extension and survival. Ann Neurol 2008;63:61-71.

9. Fragoso YD, Arruda CC, Arruda WO, et al. The real-life experience with cardiovascular complications in the first dose of fingolimod for multiple sclerosis. Arq Neuropsiquiatr 2014;72:xx-xx.

10. Min JH, Kim BJ, Lee KH. Development of extensive brain lesions following fingolimod (FTY720) treatment in a patient with neuromyelitis optica spectrum disorder. Mult Scler 2012;18:113-115. 